\title{
On the Morphology of Films Grown by Droplet-Assisted Molecular Beam Epitaxy
}

\author{
T. E. Lamas and A. A. Quivy \\ Instituto de Física da Universidade de São Paulo, Laboratório de Novos Materiais Semicondutores \\ C.P. 66318, 05315-970 São Paulo, SP, Brazil
}

Received on 23 April, 2001

\begin{abstract}
Atomic Force microscopy was used to investigate the morphology of $\mathrm{GaAs}(001)$ layers deposited by molecular beam epitaxy using a non conventional growth method that involves the supply of a few monolayers of gallium (the arsenic cell is shuttered) followed by the annealing of the surface under an arsenic flux (the gallium cell is shuttered). When the silicon shutter is opened and closed together with the gallium one, this particular growth mode allows the silicon atoms to be incorporated into the arsenic sites and p-type $\mathrm{GaAs}(001)$ layers can be obtained. Several sets of samples were grown and analyzed in order to better understand the microscopic growth mechanisms of this kind of layers and minimize the number and size of the structural defects that are characteristic of this peculiar technique.
\end{abstract}

\section{Introduction}

Since the advent of molecular beam epitaxy (MBE), beryllium (Be) has been commonly used to produce p-type GaAs layers. However, this element has several drawbacks[1] (large diffusion coefficient, segregation at high concentration, large memory effect, low purity, high toxicity) that stimulated the community to substitute it. Solid carbon has proven its suitability, usually providing doping levels up to $10^{20} \mathrm{~cm}^{-3}$ and enabling abrupt doping profiles. Another attractive option is to exploit the amphoteric characteristic of silicon (Si), with the huge advantage that both $\mathrm{n}$ and $\mathrm{p}$-type GaAs layers could be obtained in the same structure by using a single dopant. Si is the usual dopant for ntype $\mathrm{GaAs}(001)$ layers, but it has already been successfully used to produce p-type GaAs layers on high-index GaAs(N11) substrates $(\mathrm{N}=1,2,3)$ where, depending on the growth conditions (mainly the substrate temperature and V/III flux ratio), the Si atoms can enter the gallium (Ga) or arsenic (As) sites, yielding a n- or ptype GaAs layer, respectively.[2, 3] In a recent work, Quivy et al. [4] reported a new growth method that produces p-type GaAs layers using $\mathrm{Si}$ as the dopant on a $\operatorname{GaAs}(001)$ substrate which is commonly used to produce micro- and opto-electronic devices. This new doping technique is very promising because p-type layers can be obtained under normal flux conditions just by using a different sequence of the Ga, As and Si shutters. The main difference with usual growth conditions is that several monolayers (MLs) of Ga and Si are supplied without any As (the As shutter is kept closed) and then the surface is annealed under an As flux (the Ga and Si shutters are closed) to build the GaAs:Si layers. This sequence can be repeated hundreds of times to obtain thick GaAs:Si layers with a $\mathrm{p}$ character. For instance, a $0.5 \mu \mathrm{m}$-thick GaAs layer grown at $520^{\circ} \mathrm{C}$ using 180 sequences of 10MLs of Ga annealed during 15s under an As flux showed a hole density of $1.2 \times 10^{18} \mathrm{~cm}^{-3}$ for a nominal Si concentration of $4.0 \times 10^{18} \mathrm{~cm}^{-3}$. Atomicforce microscopy (AFM) measurements revealed that large structural defects were present at the surface of the film and were most probably at the origin of the low carrier mobility.

In the present work, we report on a complete AFM investigation of the surface of such samples as a function of the relevant growth parameters. This kind of study is fundamental to understand how these defects are formed, in order to be able to decrease their size and concentration and to improve the structural and electrical properties of the layers.

\section{Experimental details}

In order to better understand the structural evolution of the sample described above, a set of six samples was grown to investigate the morphology of the film at different moments of the growth and under different conditions. All the samples were grown in a Mod. Gen II MBE system from Varian on top of semi-insulating GaAs(001) substrates from Sumitomo. In the first sample, 10 MLs of $\mathrm{Ga}$ atoms were grown at $515^{\circ} \mathrm{C}$ without 
any supply of As to observe how the initial deposition of $\mathrm{Ga}$ atoms proceeds in the absence of As. The second sample was identical to the first one, except that As was supplied during $30 \mathrm{~s}$ after the Ga deposition in order to analyze the surface after a single growth cycle. The third sample consisted of a $0.25 \mu \mathrm{m}$-thick GaAs buffer grown at $515^{\circ} \mathrm{C}$ obtained by repeating 89 times the sequence of sample 2. Samples 4 and 5 were identical to samples 1 and 3 , respectively, except by the fact that they were grown at $300^{\circ} \mathrm{C}$. Finally, the sixth sample was a $0.25 \mu$ m-thick GaAs buffer grown at $515^{\circ} \mathrm{C}$ using 890 sequences consisting of $1 \mathrm{ML}$ of Ga atoms exposed during $4 \mathrm{~s}$ to the As flux. Before the growth of any of these structures, a $0.20 \mu \mathrm{m}$-thick GaAs layer was deposited at $570^{\circ} \mathrm{C}$ using conventional growth conditions to provide the same smooth surface to all the samples. As we were only concerned with the morphological characteristics of the samples, the layers were undoped (the absence of $\mathrm{Si}$ has no influence on the growth mechanisms, as will be checked later, because the dopant typically represents less than $0.01 \%$ of the total number of surface atoms). The reflection high-energy electron diffraction (RHEED) system was used to monitor the growth in situ and to determine the flux of $\mathrm{Ga}$ and As atoms that were of the order of 5.9 and $11.7 \times 10^{14}$ atoms $\cdot \mathrm{cm}^{-2} \cdot \mathrm{s}^{-1}$, respectively, for all the samples. The surface of the films was analyzed at ambient conditions with a Nanoscope IIIa AFM from Digital Instruments operating in contact mode with a sharpened silicon-nitride tip.

\section{Results and discussion}

Fig. 1 shows the topography of each sample. In Fig. 1a, one can see that the deposition of $10 \mathrm{MLs}$ of $\mathrm{Ga}$ atoms without any incident As proceeds with the formation of slightly-oval $\mathrm{Ga}$ droplets oriented along the [1-10] direction. RHEED studies actually demonstrated that the first Ga layer equally covers the whole surface, leading to the known four-fold pattern typical of a Garich surface, whereas the rest of the Ga material is accumulated in droplets spread all over the surface.[5, 6] For a given amount of material, the size and density of these droplets are a function of the adatom mobility which mainly depends, in our particular case, on the substrate temperature, as will be confirmed later. When the As flux is supplied on the Ga droplets, the specular-beam intensity of the RHEED pattern shows strong oscillations that are related to the formation of two-dimensional (2D) GaAs layers. Indeed, when the As molecules impinge on the Ga-rich surface, their sticking coefficient is maximum ( 0.5 for $\left.\mathrm{As}_{4}\right)$ and thus 2D GaAs islands are nucleated on the surface and merge to form the first GaAs layer. Once that layer is com- plete, the Ga droplets provide a fresh layer of $\mathrm{Ga}$ atoms that will react further with the new incoming As atoms. Each new 2D GaAs layer gives rise to an intensity oscillation of the specular beam that keeps oscillating until all the Ga material from the droplets has reacted. However, we can see from Fig. 1b that the new GaAs layers are not completely smooth, and structural features, also oriented along the [1-10] direction, are now
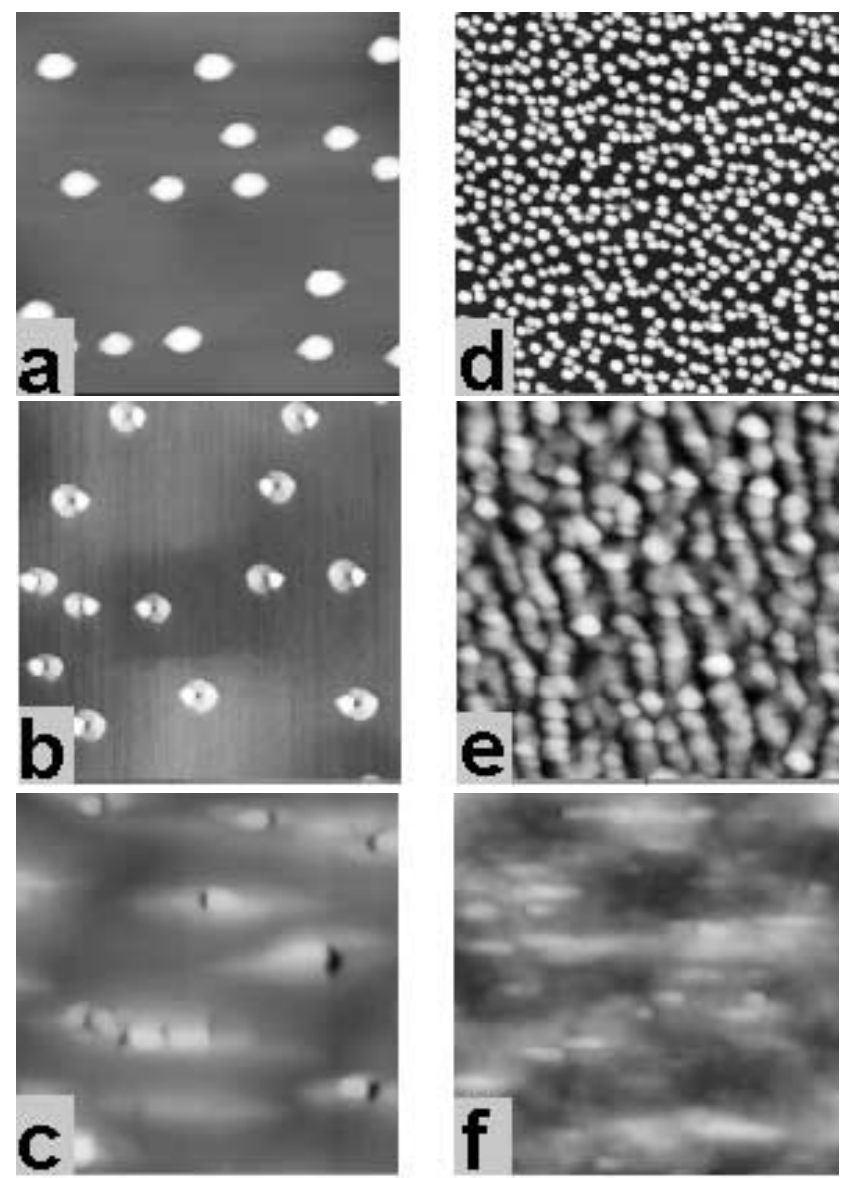

Figure 1. $2 \times 2 \mu \mathrm{m}^{2}$ AFM images of the six samples of the set. a) $10 \mathrm{MLs}$ of $\mathrm{Ga}$ grown at $515^{\circ} \mathrm{C}$ without any incident As flux; b) $10 \mathrm{MLs}$ of $\mathrm{Ga}$ grown at $515^{\circ} \mathrm{C}$ without any incident As and then exposed to As; c) a $0.25 \mu$ m-thick GaAs layer grown using 89 times the sequence described in b); d) same sample as a) but grown at $300^{\circ} \mathrm{C}$; e) same sample as c) but grown at $300^{\circ} \mathrm{C}$; f) a $0.25 \mu \mathrm{m}$-thick GaAs layer grown at $515^{\circ} \mathrm{C}$ by repeating 890 times the deposition of $1 \mathrm{ML}$ of Ga without any incident As molecules followed by the exposure of the surface to the As flux. The total grey scale and the typical height of the features observed in each image are respectively 120 and $29 \mathrm{~nm}$ in a), 50 and $13 \mathrm{~nm}$ in b), 80 and $17 \mathrm{~nm}$ in c), 50 and $13 \mathrm{~nm}$ in d), 150 and $45 \mathrm{~nm}$ in e) and 20 and $2 \mathrm{~nm}$ in $\mathrm{f}$ ).

present where the Ga droplets were previously located. These defects result from the association of two different effects: first, there is a reaction between the metallic $\mathrm{Ga}$ of the droplets and the As molecules that fall directly on them. Since the growth rate of the GaAs material is much larger at the border of the droplet than at its center (in the latter case the As molecules 
have to diffuse first through the whole thickness of the Ga droplets), there exists an accumulation of metallic $\mathrm{Ga}$ in the central region of the droplets where the second phenomenon, the melt-back etching of the GaAs buffer layer, takes place. This kind of etching occurs whenever metallic $\mathrm{Ga}$ is deposited on a GaAs surface, and its efficiency (i.e. etching rate) mainly depends on the substrate temperature.[7] Both phenomena, acting together during the annealing of the Ga droplets under the As flux, are responsible for the peculiar shape of the defects. When a $0.25 \mu \mathrm{m}$-thick GaAs layer is grown using 89 such sequences, the surface of the film shows large oval defects with small craters inside (Fig. 1c). This kind of defects is typical of Ga-rich growth conditions that are known to generate oval defects oriented along the [1-10] direction, which is consistent with our growth method and AFM results. The small apparent craters are most probably due to the melt-back etching of the underneath GaAs layers by the Ga droplets of the last cycles of the growth. The morphology of this thick GaAs layer is very similar to the one of the thick p-type Si-doped $\mathrm{GaAs}(001)$ layer grown in ref. [4] by the same method, confirming that the Si atoms do not influence the growth mechanisms and the morphology of the film.

Since the roughness of the sample is mainly due to the presence of $\mathrm{Ga}$ droplets, a reduction of the surface corrugation height would be expected when smaller (or no) Ga droplets are present on the surface. That can be achieved by depositing a smaller number of Ga layers per cycle or by adopting a lower substrate temperature during Ga deposition. At first glance, a lower growth temperature has several advantages: the Ga droplets are more homogeneous, smaller and more numerous (Fig. 1d), due to the smaller diffusion length of the $\mathrm{Ga}$ adatoms. In addition, the melt-back etching is less efficient at lower temperature, decreasing considerably the depth of the craters. Unfortunately, when the As flux is allowed on the Ga droplets at such a low temperature, the growth of the GaAs layers is very rough and the total corrugation height is larger than at higher temperature (Fig. 1e). As a consequence the best way to reduce the roughness of the film is using a larger number of cycles involving the deposition (at intermediate temperature) of a smaller number of $\mathrm{Ga}$ layers without any incident As. The growth at $515^{\circ} \mathrm{C}$ of a $0.25 \mu \mathrm{m}-$ thick GaAs layer consisting of 445 cycles of $2 \mathrm{MLs}$ of Ga exposed to As during 8s showed that the size of the oval defects and craters were much smaller. The limit of this growth method is clearly achieved when a single Ga layer is deposited and then annealed in the presence of the As flux. In this extreme case, no Ga droplets are formed because the first (and unique) $\mathrm{Ga}$ layer is homogeneously spread over the whole sample and reacts with the As-rich surface, as confirmed by the change of the usual two-fold RHEED pattern into a clear four-fold pattern typical of flat Ga-rich conditions. Since there are no droplets on the surface at any step of the growth cycle, there are no oval defects and no melt-back etching responsible for the formation of the craters. In these conditions the roughness of the surface is minimum, as can be seen in Fig. 1f.

\section{Conclusion}

We presented AFM results about the surface morphology of samples obtained by droplet-assisted MBE, a growth technique that was recently employed to produce p-type $\operatorname{GaAs}(001)$ layers using Si as the dopant. At the initial stage, the deposition of several MLs of Ga without any incident As flux proceeds with the formation of $\mathrm{Ga}$ droplets on the surface. When the As flux is admitted, the $\mathrm{Ga}$ droplets react with the incoming As molecules and form 2D GaAs layers, leaving behind them structural defects and small craters due to the melt-back etching of the underneath GaAs layers by the Ga droplets. Thick GaAs layers can be obtained by repeating this sequence, but the final roughness of the film was minimized when a single Ga layer was deposited at a time and then annealed under the As flux. The optimization of the structural properties of such layers is very important to the device industry in order to be able to obtain both $\mathrm{n}$ and p-type $\operatorname{GaAs}(001)$ layers with a single element $(\mathrm{Si})$ using the same growth conditions but different shutter sequences.

\section{Acknowledgments}

We would like to thank FAPESP for financial support (grants 99/10510-7, 99/01225-7 and 98/14489-0) and the "Laboratório de filmes finos do IFUSP" for the SPM facility (FAPESP proc. 95/05651-0).

\section{References}

[1] M. Takahashi, M. Hirai, K. Fujita, N. Egami and K. Iga, J. Appl. Phys. 82, 4551 (1997).

[2] K. Agawa, K. Hirakawa, N. Sakamoto, Y. Hashimoto and T. Ikoma, Appl. Phys. Lett. 65(9), 1171 (1994).

[3] A. A. Quivy, M. Frizzarini, A L. Sperandio, E. C. F. da Silva, J. R. Leite, Brazilian J. Physics 27/A, 125 (1997).

[4] A.A. Quivy, A.L. Sperandio, E.C.F. da Silva and J.R. Leite, J. Crystal Growth 206, 171 (1999).

[5] J.H. Neave, B.A. Joyce and P.J. Dobson, Appl. Phys. A 34, 179 (1984).

[6] T. Suzuki and T. Nishinaga, J. Crystal Growth 142, 61 (1994).

[7] K. Kishino, S. Kinoshita, S. Konno, and T. Tako, Jap. J. Appl. Phys. 22, L473 (1983). 